\title{
PEGylated Gold Nanoparticles as a Biocompatible Contrast Agent in X-ray Imaging of Breast Tumor in Mouse Model
}

\section{MOJTABA Salouti ${ }^{\star 1}$, ZAHRA Heidari ${ }^{1}$, FARANAK Saghatchi ${ }^{2}$}

\author{
${ }^{1}$ Biology Research Center, Zanjan Branch, Islamic Azad University, Zanjan, Iran. \\ ${ }^{2}$ Department of Radiology, Faculty of Paramedical and Health Sciences, Zanjan University of \\ Medical Sciences, Zanjan, Iran.
}

ARTICLE INFO

Article No.: 0511014228

Type: Research

DOI: 10.15580/GJBS.2014.5.0511014228

Submitted: $11 / 05 / 2014$

Accepted: 07/07/2014

Published: 17/09/2014

${ }^{\star}$ Corresponding Author

Mojtaba Salouti

E-mail: saloutim@yahoo.com

Phone: (+98) 2414224024

Keywords:

Breast Cancer; X-ray Imaging;

Contrast Agent; Gold Nanoparticles;

PEGylation
Objective: Though biopsy is ultimately the gold standard for breast cancer detection, medical imaging can be a valuable tool for gathering information in vivo for both screening and diagnosis. In this study, we propose the use of gold nanoparticles (GNPs) modified with polyethylene glycol (PEG) as a biocompatible contrast agent in $\mathrm{X}$ - ray imaging of breast cancer in mouse model.

Methods: Gold nanoparticles in spherical shape were coated with PEG and the interaction was investigated by UV/vis spectroscopy. The UV/vis analysis was performed to check the stability of PEG-GNPs complex in human blood serum. The biodistribution and $X$-ray imaging studies were performed to evaluate the localization of PEGylated GNPs in xenografted breast tumor in mouse model.

Results: The interaction of PEG with GNPs was confirmed by UV-vis spectroscopy. The stability of PEG-GNPs was found high at human blood serum with no aggregation. The biodistribution study showed the accumulation of PEGylated GNPs in the site of tumor. The tumor was properly visualized in X-ray images in mouse model.

Conclusions: The results showed the potential of PEGylated gold nanoparticles as a contrast agent for X-ray imaging of breast cancer in humans that needs further investigations. 


\section{INTRODCUTION}

Breast cancer is the most frequently diagnosed solid malignancy in women in the world (Heidari and Salouti, 2012). Advances in diagnosis of breast cancer lead to decline in mortality and morbidity (James et al., 2007). Many efforts have ever been exerted to the new techniques to enable us collect more information by noninvasive methods. These techniques such as magnetic resonance imaging (MRI), computed tomography (CT) and mammography minimize the use of breast biopsy in women who do not have breast cancer (Gunasekera and Pankhurst, 2009). The recent concerns on the toxicity of commonly used contrast agents such as gadolinium for MRI and iodine compounds for radiography and CT justify the need for less toxic contrast agents. Current $X$-ray contrast agents (tri-iodobenzene) impose serious limitations on medical imaging which include: short imaging times, the need for catheterization in many cases and occasional renal toxicity (Gunasekera and Pankhurst, 2009).

Nanomaterials have become increasingly important in the development of new contrast agents for in vivo imaging (Hutter and Maysinger, 2010). Gold nanoparticles have remarkable advantages as an effective X-ray contrast agent such as negligible osmolality of $7.2 \mathrm{mM}$, (Sungsook et al., 2013), low viscosity (Sungsook et al., 2013), higher absorption coefficient than iodine, and less interference of bones and tissues (Sungsook et al., 2013; Hainfeld and Slatkin, 2006). Compared with low-molecular-weight iodine solutions, GNPs with low toxicity can remain in the blood longer, benefiting prolonged imaging time (Rabin and Perez, 2007; Hainfeld and Slatkin, 2006). GNPs have a higher X-ray absorption than iodine around $100 \mathrm{keV}$ and at low energies $(<30 \mathrm{keV})$, which is a useful energy range for clinical CT, fluoroscopy, and mammography (Rabin and Perez, 2007; Hainfeld and Slatkin, 2006). Gold nanoparticles, between 10 and $100 \mathrm{~nm}$ in size, preferentially accumulate at the tumor sites because of hallmarks of tumors such as the fenestrated vasculature and poorly lymphatic drainage, resulting in an enhanced permeability and retention (EPR) effect (De Jong and Hagens, 2008; Natalie et al., 2007). Hainfeld and Slatkin (2006) used free gold nanoparticles for imaging of EMT6 murine breast tumor in animal model for the first time (Hainfeld and Slatkin, 2006). Modifying the surface of GNPs with polyethylene glycol has been found to elongate the circulation time of nanoparticles in blood by hiding particles from the reticuloendothelial system in liver and lungs (Niidome and Yamagata, 2006). De Jong and Hagens reported that the EPR effect along with longer circulation time, achieved by GNPs PEGylation, can increase concentrations of modified gold nanoparticles in tumors by $10-100$-fold in comparison with free GNPs (De Jong and Hagens, 2008; Natalie et al., 2007). In this study, we modified gold nanoparticles with polyethylene glycol to make it biocompatible and performed biodistribution and imaging studies to investigate the potential application of PEGylated GNPs in X-ray imaging of breast cancer as a contrast agent in mouse model. This report is the first, to our knowledge, to use PEG modified gold nanoparticles as a contrast agent in X-ray imaging of breast cancer.

\section{MATERIAL AND METHODS}

\section{Materials}

Gold nanoparticles in the size of $10 \mathrm{~nm}$ with spherical shape $(\lambda$ max: $540 \mathrm{~nm})$ were purchased from Sigma Aldrich, USA. Polyethylene glycol (mPEG-SH 5000) and HEPES buffer were purchased from Merck, Germany. BALB/c mice (8-10 weeks old and 25-35g weight) were purchased from Pasteur Institute, Tehran, Iran. All the glass wares were first treated with aqua regia, rinsed with deionized water and then oven-dried.

\section{Surface Modification of Gold Nanoparticles}

PEG-modified gold nanoparticles were prepared by mixing mPEG-SH with gold nanoparticles stabilized by citrate (Niidome and Yamagata, 2006). Three $\mathrm{ml}$ of 10 $\mathrm{nm}$ GNPs $(\mathrm{OD}=3)$ was mixed with $212 \mu \mathrm{l}$ of PEG-thiol solution $(0.5 \mu \mathrm{g} / \mu \mathrm{l})$ in a round-bottom flask, and stirred for $2 \mathrm{~h}$ at room temperature. Then, the PEGylated gold nanoparticles were purified by repeated centrifugation (9600 $\mathrm{g}$ for $30 \mathrm{~min}, 3$ times), and the GNPs pellet was redissolved in $2 \mathrm{ml}$ of phosphate buffer saline (PBS) at pH 7.4 after removing the supernatant (Wang et al., 2011; Huang and El-Sayed, 2006, 2010; Eghtedari and Liopo, 2009; Popovtzer and Agrawal, 2008; Niidome and Yamagata, 2006). The purified PEGylated GNPs solution was stored at $4^{\circ} \mathrm{C}$.

\section{PEGylated Gold Nanoparticles Characterization}

UV-vis spectrophotometer (CARY-100 BIO, Varian, USA) was used for investigating the interaction of PEG with gold nanoparticles (Huang and El-Sayed, 2006, 2010; Eghtedari and Liopo, 2009; Niidome and Yamagata, 2006). The spectrum ranged from 400 to 700 $\mathrm{nm}$ at the resolution of $1 \mathrm{~nm}$. The plasmon band at 540 $\mathrm{nm}$ for the wine red colloidal gold in UV-vis spectrum is a characteristic of gold nanoparticles. The shift of surface plasmon resonance band (SPR) to a longer wavelength shows the capping of GNPs surface with PEG molecules (Huang and El-Sayed, 2006, 2010; Eghtedari and Liopo, 2009; Niidome and Yamagata, 2006).

\section{In vitro Blood Serum Stability}

$100 \mu \mathrm{l}$ of PEGylated GNPs solution was added to one $\mathrm{ml}$ of human blood serum sample. The optical absorbance 
of solution was monitored at $545 \mathrm{~nm}$ at multiple time points up to $24 \mathrm{~h}$ (Kircher et al., 2012; Eghtedari and Liopo, 2009; Gokce et al., 2004; Fura et al., 2003). The control solution was included in the human blood serum and in $100 \mu \mathrm{l}$ of PBS only (1/1 ratio) (Kircher et al., 2012). The samples were transferred to a $1 \mathrm{~cm}$ cuvette. The light absorption of samples was determined using a UV/vis spectrophotometer. Subtracting the "serum" absorbance from "the PEG-GNPs in serum" absorbance shows PEG-GNPs absorbance. If PEG-GNPs solution has high stability, the absorption peak remains intact around $545 \mathrm{~nm}$ (Kircher et al., 2012; Eghtedari and Liopo, 2009).

\section{Breast Tumor Xenograft}

Breast tumor was established by subcutaneous implantation of spontaneous tumor fragments $(3 \times 3 \times 3$ $\mathrm{mm}$ ) in the left side of the abdominal region of normal inbred female BALB/c mice (Salouti and Babaei, 2010). Biodistribution and imaging studies were performed when the xenograft volume reached $100 \mathrm{~mm}^{3}$. All the animal experiments were approved by the Animal Care Committee of Tarbiat Modares University, Tehran. Iran.

\section{Biodistribution Study}

For biodistribution assessment, 4 groups of 5 BALB/C mice with breast tumor were injected with $200 \mu$ of PEG modified GNPs solution at the concentration of $2.7 \mathrm{~g} \mathrm{~kg}^{-1}$ GNPs via the tail vein (Ganeshchandra and Keishiro, 2008; Ding and Chen, 2008). At 1, 2, 4, 6, 8, 12 and $24 \mathrm{~h}$ post injection, the mice were sacrificed using $\mathrm{CO}_{2}$ gas. The liver, heart, kidney, small and large intestines, stomach, muscle, blood and tumor were dissected and weighted. The tissues were lysed in a $15 \mathrm{ml}$ tube with aqua regia and subjected to the quantitative analysis of PEGylated GNPs by atomic absorption spectroscopy (Ganeshchandra and Keishiro, 2008; Ding and Chen, 2008). The data was represented as a percentage of injected dose per gram of each tissue $(\% / D / g)$.

\section{X-ray Imaging}

For X-ray imaging, 5 BALB/c mice with breast tumor were injected with $200 \mu \mathrm{l}$ of PEGylated GNPs solution at the concentration of $2.7 \mathrm{~g} \mathrm{~kg}^{-1}$ GNPs via the tail vein (Hainfeld and Slatkin, 2006). Prior to X-ray imaging, the animals were anesthetized with the combination of xylazine hydrochloride and ketamine hydrochloride. The imaging was performed using a clinical mammography unit (company PAYAMED, model MAMMO X-RAY UNIT $100 \mathrm{KHZ}$ ) at $4 \mathrm{~h}$ post-injection of the produced contrast agent (22 Kv and $10 \mathrm{mAs}$ ) (Hainfeld and Slatkin, 2006).

\section{Statistical Analysis}

All the experiments were repeated 5 times and the results were expressed as mean $\pm S D$. Anova test was used to compare the accumulation of PEG modified GNPs solution in vital organs and tumor tissue of mouse model in different times ( $p$-value< 0.05).

\section{RESULTS}

\section{PEG modified Gold Nanoparticles Characterization}

The absorption spectrum of PEG modified gold nanoparticles solution was measured after reaction of thiol group of PEG with the surface of gold nanoparticles. The UV-vis spectrum of the PEGylated GNPs showed a shift of $5 \mathrm{~nm}$ in the absorbance peaks between free GNPs and PEG-modified GNPs (Figure 1). The shift in the spectrum indicated that the PEG molecules have successfully replaced the citrate anions and bound to the GNPs surfaces (Huang and El-Sayed, 2006, 2010; Eghtedari and Liopo, 2009; Popovtzer and Agrawal, 2008; Niidome and Yamagata, 2006). No aggregation happened in PEGylated GNPs solution up to 6 months storage at $4{ }^{\circ} \mathrm{C}$ (no observable change in the solution color) that showed the high stability of PEG modified gold nanoparticles. 


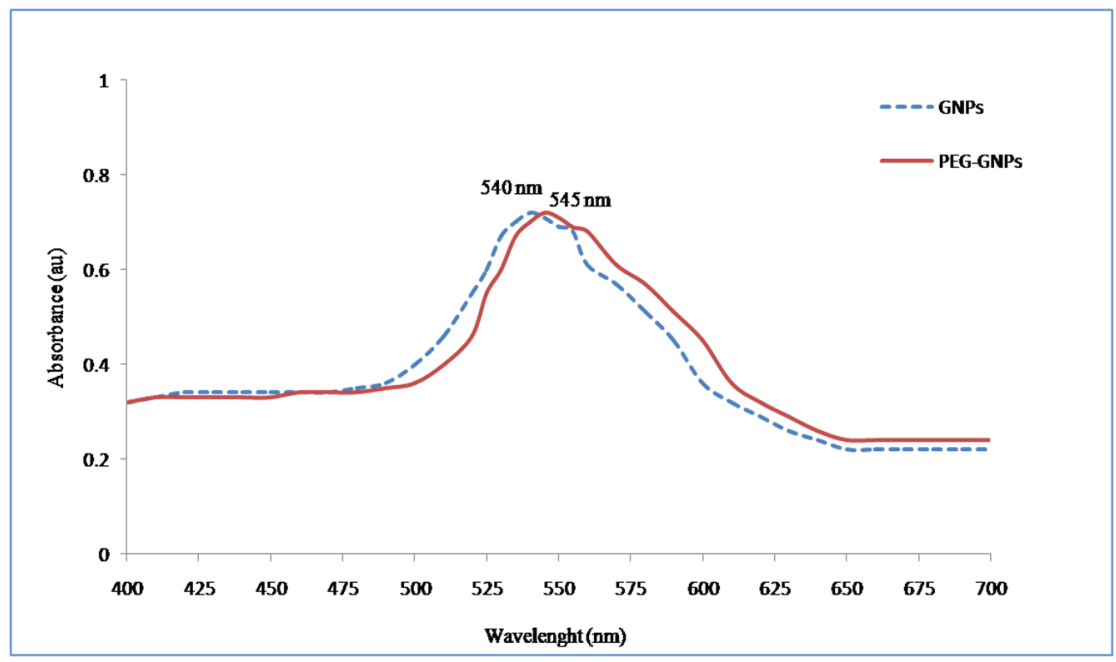

Figure 1: UV-vis spectra of (a) free GNPs and b) PEG-GNPs solution. The appearance of a new peak at $545 \mathrm{~nm}$ is due to the coating of gold nanoparticles with PEG

\section{In vitro Blood Serum Stability}

Figure 2 depicts the light absorption of the PEGylated GNPs as a function of time after being in human blood serum up to $24 \mathrm{~h}$. The blue (the absorbance of serum) and red (the absorbance of serum plus PEG-GNPs) curves show a slight increase of absorbance over time, an effect which is likely due to the samples water evaporation, thereby increasing PEGylated GNPs concentration. The green curve, representing the subtraction of "serum" from "serum plus PEGylated GNPs", shows no aggregation of PEGylated GNPs solution when mixed with human blood serum. This study confirmed the stability of PEGylated GNPs within serum microenvironment. PEG-modification was expected to reduce unspecific binding with serum components such as serum proteins.

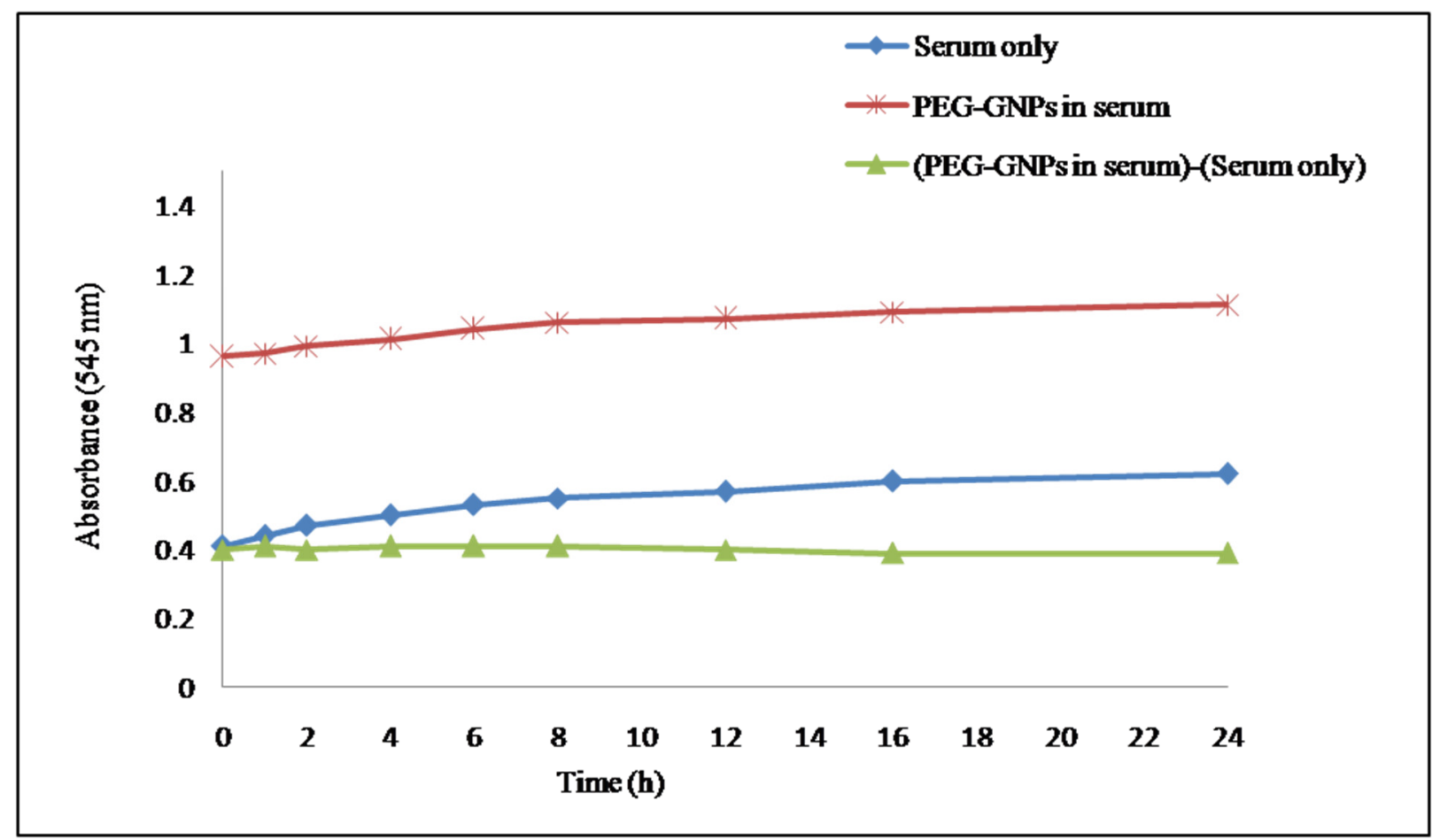

Figure 2: Optical serum stability of PEG-GNPs. The PEG-GNPs showed a high level of optical stability when exposed to serum 


\section{Biodistribution Study}

Figure 3 shows the biodistribution of PEGylated GNPs in $\mathrm{BALB} / \mathrm{c}$ mice bearing breast tumor at $1,2,4,6,8,12$ and $24 \mathrm{~h}$ after IV administration expressed as percentage of injected dose per gram of each organ
(\%ID/g). The analysis of biodistribution study showed that PEG modified gold nanoparticles clears from the blood slowly, permitting longer times for imaging. The retention in liver was low with elimination by the kidneys. The results showed the localization of new preparation at the site of tumor ( $p$-value $<0.05)$.

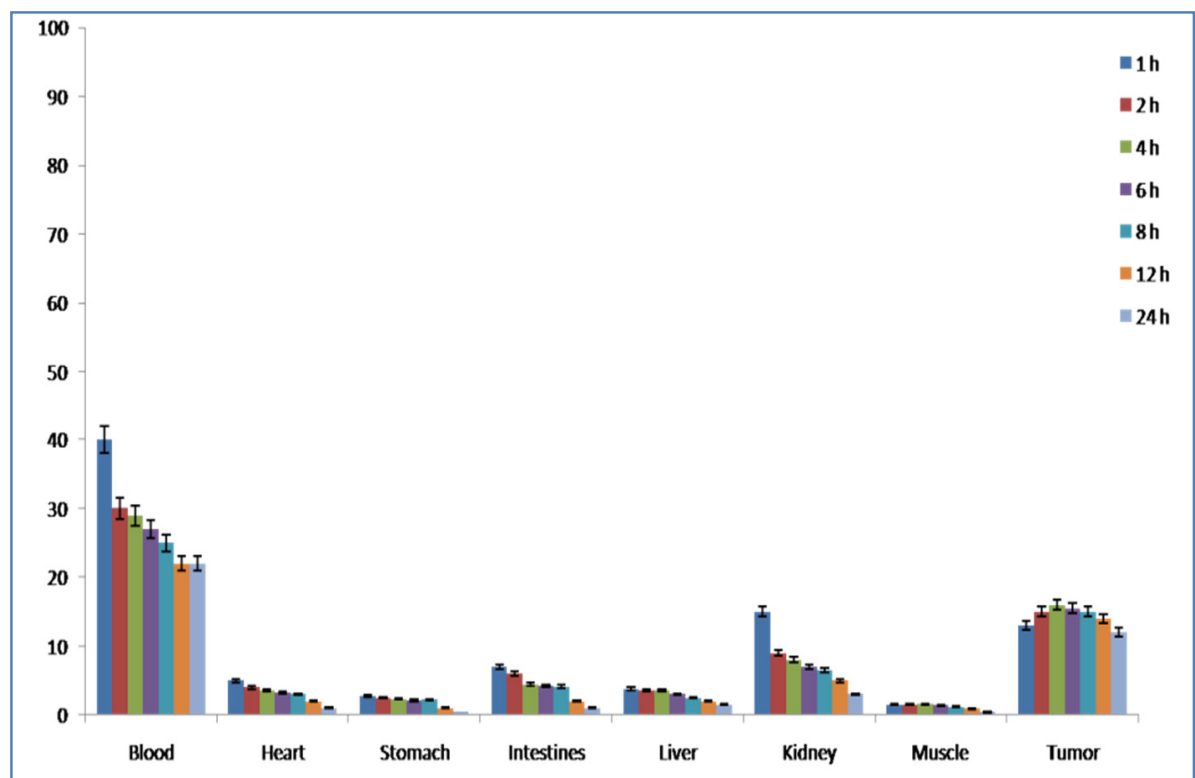

Figure 3: Biodistribution data in vital organs of BALB/c mice bearing breast tumor at time intervals of 1, 2, 4, 6, 8, 12 and $24 \mathrm{~h}$ post injection of PEG-GNPs solution. This figure shows accumulation of PEG-GNPs solution in the tumor in all the measured times specially at $4 \mathrm{~h}$ post injection $(n=5)(p<0.05)$

\section{X-ray Imaging}

The tumor was visualized properly as early as $4 \mathrm{~h}$ after injection of PEGylated GNPs as a contrast agent. X-ray image of a representative BALB/c mice with breast tumor, $4 \mathrm{~h}$ after administration of the new contrast agent, has been shown in figure 4 . 

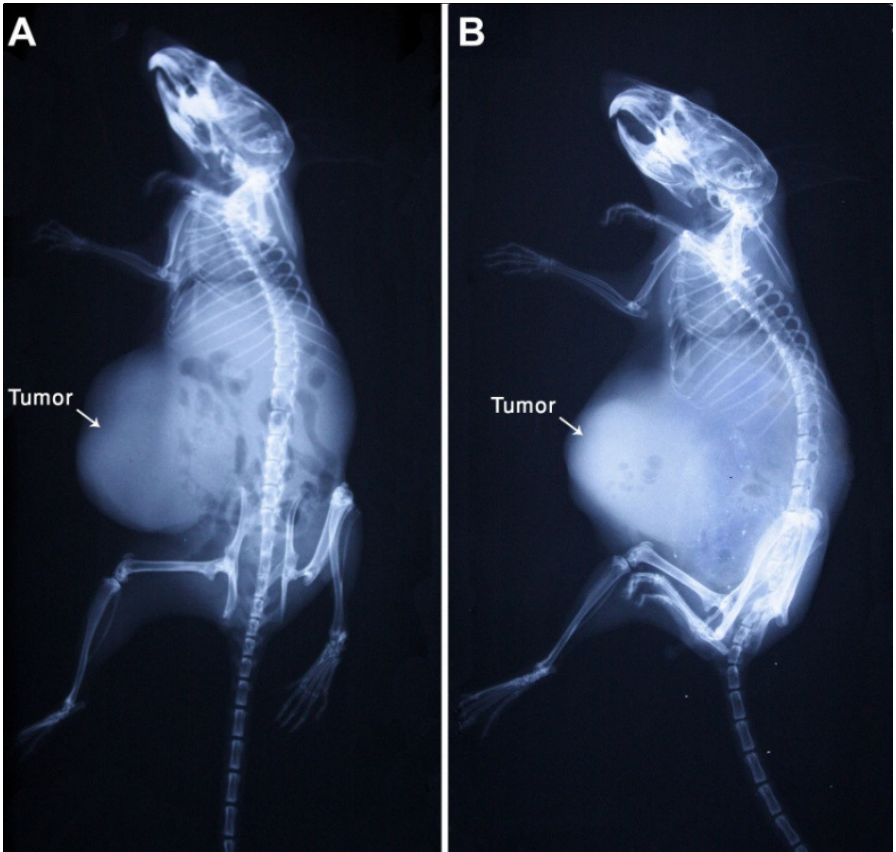

Figure 4: X-ray image of a representative BALB/C mice bearing breast tumor before injection $(A)$ and 4 $h$ after injection of PEGylated GNPs

\section{DISCUSSION}

X-ray imaging, is among the most convenient imaging/diagnostic tools today in terms of availability, efficiency and cost (Chanda and Kattumuri, 2010; Tong and Wei, 2009). Gold nanoparticles are increasingly becoming the subject of investigation as radiographic contrast media due to the toxicity of most commonly used contrast agents (Hutter and Maysinger, 2010). However, unmodified GNPs have some disadvantages like cell aging and low blood circulation time (Mironava et al., 2013). In this study, we successfully modified citrate functionalized gold nanoparticles with PEG to overcome these problems, as a contrast agent for X-ray imaging of breast tumor in mouse model.

Chonn et al. reported that high affinity of gold nanoparticles to blood proteins can rapidly reduce its circulation time in blood stream (Kairemo and Erba, 2008; Brigger et al., 2002). The citrate anions that coat the surface of nanoparticles impart a negative surface charge to the GNPs that would promote the non-specific attachment of serum proteins in vivo (Kairemo and Erba, 2008; Brigger et al., 2002). This would severely hinder the ability of GNPs to reach the tumor site. The GNPs are also stabilized through mutual electrostatic repulsion between neighboring negatively charged nanoparticles (Kairemo and Erba, 2008; Brigger et al., 2002). This repulsion can be shielded by ions present in blood that would result in the aggregation of GNPs (Kairemo and Erba, 2008; Brigger et al., 2002). One effective solution to prevent opsonization and enable nanoparticles to circulate for a long time in blood microenvironment is to construct a 'stealth-shielding' layer composed of watersoluble polymers (with low specificity for human blood proteins) on the surfaces of gold particulates (Niidome and Yamagata, 2006). It is suggested that polyethylene glycol helps nanoparticles to escape from the reticuloendothelial system by steric repelling of the opsonin proteins that are essential for the clearance of nanoparticles from blood stream (Niidome and Yamagata, 2006).

High stability of the PEGylated GNPs complex within blood serum microenvironment is a key issue for in vivo studies (Eghtedari and Liopo, 2009). The stability study in human blood serum demonstrated the high stability of PEGylated GNPs up to $24 \mathrm{~h}$.

The biodistribution study at different times showed the increasing uptake of PEGylated GNPs at tumor tissue by the time up to $4 \mathrm{~h}$. The results indicated that PEG-modification of gold nanoparticles dramatically changes biodistribution of GNPs in blood in comparison with free GNPs. The findings showed that PEGylated GNPs were stably circulating in blood until $24 \mathrm{~h}$ after injection and clear slowly from blood that provides longer imaging times. The results also showed low retention of PEGylated GNPs complex in liver and the elimination of PEG-modified GNPs by kidneys. These results demonstrated that $\mathrm{X}$-ray imaging can be taken as early as $4 \mathrm{~h}$ after injection of the produced contrast agent. The xenografted breast tumor was visualized in X-ray images; $4 \mathrm{~h}$ post injection of PEGylated GNPs complex as a contrast agent with good clarity in mouse model. This finding was in accordance with the data collectedresu in biodistribution study. 
One may consider that PEG-modified GNPs can be used for X-ray imaging of other cancers because of its uptake mechanism. The application of PEG-GNPs contrast agent for $\mathrm{X}$-ray imaging can have a significant impact on health care, owing to the ubiquitous nature of $\mathrm{X}$-ray in clinical setting along with development of hybrid systems like SPECT-CT.

\section{CONCLUSION}

The results showed that PEGylated GNPs can be considered as a promising candidate in X-ray imaging of human breast cancer as a contrast agent which needs further investigations.

\section{ACKNOWLEDGMENT}

We thank Zanjan Branch, Islamic Azad University for valuable support.

\section{FINANCIAL DISCLOSURE}

Authors have no relevant financial interests related to the materials in the manuscript.

\section{THE ROLE OF SPONSORS}

The funding organizations are public institutions and had no role in the design and conduct of the study; collection, management, and analysis of the data; or preparation, review, and approval of the manuscript.

\section{FUNDING}

This study was financially supported by Biology Research Center, Zanjan Branch, Islamic Azad University, Zanjan, Iran.

\section{AUTHORS' CONTRIBUTION}

Mojtaba Salouti developed the original idea and the protocol, abstracted and analyzed data, revised the manuscript, and is the guarantor. Zahra Heidari and Faranak Saghatchi performed the experiments including $\mathrm{X}$-ray imaging and wrote the preliminary manuscript.

\section{DECLARATION OF INTEREST}

The authors declare that there is no conflict of interest that could be perceived as prejudicing the impartiality of the research reported.

\section{REFERENCES}

Brigger I, Dubernet C and Couvreur P (2002). Nanoparticles in cancer therapy and diagnosis. Advanced Drug Delivery Reviews. 54: 631-651.

Chanda N and Kattumuri V (2010). Bombesin functionalized gold nanoparticles show in vitro and in vivo cancer receptor specificity. PNAS. 107: 87608765.

De Jong W and Hagens W (2008). Particle sizedependent organ distribution of gold nanoparticles after intravenous administration. Biomaterials. 29: 1912-1919.

Ding A and Chen Y (2008). HER-2 Antibody Conjugated Gold NanoRod particles for in vivo Photothermal Therapy. IEEE. 882-885.

Eghtedari M and Liopo AV (2009). Engineering of Hetero-Functional Gold Nanorods for the in vivo Molecular Targeting of Breast Cancer Cells. NANO LET. 9: 287-291.

Fura A, Harper TW, Zhang $\mathrm{H}$, Fung $\mathrm{L}$ and Shyu WC (2003). Shift in $\mathrm{pH}$ of biological fluids during storage and processing: effect on bioanalysis. Journal of Pharmaceutical and Biomedical Analysis. 32(3):513522.

Ganeshchandra S and Keishiro T (2008). Biodistribution of colloidal gold nanoparticles after intravenous administration: Effect of particle size. Colloids and Surfaces B: Biointerfaces. 66: 274-280.

Gokce G, Citil M, Gunes V, and Atalan G (2004). Effect of time delay and storage temperature on blood gas and acid-base values of bovine venous blood. Research in Veterinary Science. 76(2):121-127.

Gunasekera UA and Pankhurst QA (2009). Imaging applications of nanotechnology in cancer. Targ Oncol. 4:169-181.

Hainfeld J F and Slatkin D N (2006). Gold nanoparticles: a new X-ray contrast agent, The British Journal of Radiology. (79) 248-253.

Heidari Z and Salouti M (2012). Targeting Molecular Imaging of Breast Cancer by Radioimmunodetection Method in Nuclear Medicine. Current Molecular Imaging. 1: 26-43.

Huang X and El-Sayed I (2006). Cancer Cell Imaging and Photothermal Therapy in the Near-Infrared Region by Using Gold Nanorods. J. AM. CHEM. SOC. 128: 2115-2120.

Huang X and El-Sayed M (2010). Gold nanoparticles: Optical properties and implementations in cancer diagnosis and photothermal therapy. Journal of Advanced Research. 1: 13-28.

Hutter E and Maysinger D (2010). Gold Nanoparticles and Quantum Dots for Bioimaging. Microscopy Research and Technique. 1-13.

James X., Jie Z. and Jing Y., 2007. Gold-based nanoparticles for breast cancer diagnosis and treatment. IEEE. 2882-2885.

Kairemo K and Erba P (2008). Nanoparticles in Cancer. Current Radiopharmaceuticals. 1: 30-36. 
Kircher MF, Zerda A and Jokerst JV (2012). Brain tumor molecular imaging strategy using a new triplemodality MRI-photoacoustic-Raman nanoparticle. NAT MED. 18: 829-834.

Mironava T, Hadjiargyrou M, Simon M and Rafailovich M (2013). Gold nanoparticles cellular toxicity and recovery: Adipose derived stromal cells. Nanotoxicology. 1: 769128.

Natalie P, Praetorius TK and Mandal (2007). Engineered Nanoparticles in Cancer Therapy. Recent Patents on Drug Delivery \& Formulation. 1: 37-51.

Niidome T and Yamagata M (2006). PEG-modified gold nanorods with a stealth character for in vivo applications. Journal of Controlled Release. 114: 343-347.

Popovtzer R and Agrawal A (2008). Targeted Gold Nanoparticles Enable Molecular CT Imaging of Cancer. NANO LET. 8(12): 4593-459.
Rabin O and Perez JM (2007). An X-ray computed tomography imaging agent based on long-circulating bismuth sulphide nanoparticles, nature materials, 6 : 118-122.

Salouti M., Babaei M.H., 2010. Comparison of 99mTclabeled PR81 and its $F(a b 0) 2$ fragments as radioimmunoscintigraphy agents for breast cancer imaging. Ann Nucl Med. 1-6

Sungsook A, Sung YJ and Sang JL (2013). Review: Gold Nanoparticle Contrast Agents in Advanced Xray Imaging Technologies, Molecules. 18: 58585890.

Tong L and Wei Q (2009). Gold nanorods as contrast agents for biological imaging: optical properties, surface conjugation, and photothermal effects. Photochem Photobiol. 85(1):1- 21.

Wang J, Liuand D and Wang Z (2011). Synthesis and cell-surface binding of lectin-gold nanoparticle conjugates, The Royal Society of Chemistry. 